CASE REPORT

\author{
J.M. Hoxworth \\ A.C. Patel \\ E.P. Bosch \\ K.D. Nelson
}

\section{Localization of a Rapid CSF Leak with Digital Subtraction Myelography}

SUMMARY: A 53-year-old woman with superficial siderosis underwent spinal MR imaging, which demonstrated a large cervicothoracic epidural fluid collection compatible with a CSF leak. Conventional and dynamic CT myelography failed to localize the dural tear because of rapid equilibration of myelographic contrast between the thecal sac and the extradural collection. The superior temporal resolution of digital subtraction myelography precisely localized the CSF leak preoperatively and led to the successful surgical correction of the dural tear.
0 ne limitation of CT myelography to identify a CSF leak is temporal resolution. During the time elapsed from intrathecal contrast injection under fluoroscopy to acquisition of the CT images, equilibration of contrast between intradural and extradural CSF collections occurs. When the extradural CSF collection is large, localizing the responsible dural tear can be challenging as a result. Dynamic CT myelography has been used to overcome this problem ${ }^{1}$ but still has temporal limitations inherent to the volume of tissue that must be imaged. We describe the case of a patient in whom chronic bleeding from a thoracic dural tear was the presumptive cause of superficial siderosis. The site of the tear was not adequately defined by myelography, postmyelography CT, or dynamic CT myelography, but digital subtraction myelography provided accurate preoperative localization.

\section{Case Report}

A 53-year-old woman with progressive impaired balance, hearing loss, lower extremity numbness, and chronic daily headaches for the past 5 years underwent neurologic evaluation at our institution. On physical examination, the salient abnormalities included brisk reflexes in both upper and lower limbs, mute plantar responses, asymmetric impairment to light-touch and pin-prick sensation in both legs below the groin, prominent dysmetria on heel-to-shin testing, a widebased ataxic gait, and positive Romberg test. An audiogram confirmed right-greater-than-left sensorineural hearing loss. Tibial somatosensory-evoked responses revealed a prolonged lumbar-scalp interpeak latency suggesting a central conduction delay. The results of an atraumatic lumbar puncture included 1+ xanthochromia, 48 red blood cells per microliter, elevated protein of $122 \mathrm{mg} / \mathrm{dL}$, and hemosiderin-containing macrophages.

MR imaging of the brain and entire spine revealed prominent T2 shortening along the surface of the cerebellum, brain stem, and spinal cord consistent with susceptibility from hemosiderin deposition. A nonenhancing ventral epidural spinal fluid collection extended from the inferior aspect of $\mathrm{C} 4$ through the T6-T7 disk space (Figs $1 A,-B$ ). Moreover, a T2 hypointense fluid-fluid level suggestive of blood products was layering dependently in the distal thecal sac (not shown).

Results of 3D time-of-flight head MR angiography were unremarkable. Because gadolinium-enhanced MR angiography of the

Received July 6, 2008; accepted after revision July 22

From the Departments of Radiology (J.M.H., A.C.P., K.D.N.) and Neurology (E.P.B.), Mayo Clinic, Scottsdale, Ariz.

Please address correspondence to Dr. Joseph M. Hoxworth, Mayo Clinic, Neuroradiology Section, Department of Radiology, 13400 E Shea Blvd, Scottsdale, AZ 85259-5404; e-mail: hoxworth.joseph@mayo.edu

DOI 10.3174/ajnr.A1294 spine suggested mildly prominent and serpiginous vessels along the dorsal aspect of the distal cord, the patient underwent catheter spinal angiography, the results of which were negative.

Because the patient's presumptive diagnosis was superficial siderosis secondary to bleeding from a dural tear, a routine myelogram was performed via lumbar puncture, and postmyelography CT of the entire spine was obtained (Figs $1 C,-D$ ). The diffuse contrast opacification of the ventral epidural fluid collection confirmed the presence of a CSF leak. Subsequently, a dynamic CT myelogram was performed (not shown) by use of previously published methodology, ${ }^{1}$ but the site of the dural tear was not adequately localized because the rapid nature of the CSF leak resulted in a significant amount of contrast extending within the epidural fluid collection over 5 vertebral levels (T2-T6).

For maximization of temporal resolution, a digital subtraction myelogram was undertaken. The patient was placed in the prone position in the biplane angiography suite (Axiom Artis dBA; Siemens Medical Solutions, Malvern, $\mathrm{Pa}$ ), and foam padding was placed beneath her pelvis to elevate and flatten the lumbar lordosis relative to the thoracic kyphosis. Fluoroscopic-guided lumbar puncture was performed via the L2-L3 interlaminar space by use of a 20-gauge, 8.9-cm spinal needle. With suspended respiration, a digital subtraction acquisition ( 5 frames per second) encompassing C7-T7 was performed in the lateral projection during the intrathecal injection of 10 mL Omnipaque-300 (GE Healthcare, Piscataway, NJ) at a rate of approximately $1 \mathrm{~mL} / \mathrm{s}$ by hand. The superb temporal resolution allowed clear visualization of the CSF leak at the site of a T5-T6 partially calcified disk extrusion (Fig 2). No additional communications between the thecal sac and epidural fluid collection were identified.

Surgical exploration was performed with T4-T7 laminectomies and right $\mathrm{T} 5$ costotransversectomy with $\mathrm{T} 5$ nerve root sacrifice to mobilize the thecal sac. A large amount of posterior longitudinal ligament venous tissue was adherent to connective tissue over the dura and seemed to be the source of bleeding into the epidural cavity filled with CSF (ultimately communicating with the subarachnoid space via the dural tear). Coagulation and resection of this segment of the posterior longitudinal ligament resulted in cessation of bleeding. A ventral dural tear was identified near the midline at T5-T6, and this was repaired surgically by entering the thecal sac via a dorsal incision and mobilizing the cord laterally. Thoracic spine MR imaging was performed 3 months postoperatively, and the previously seen ventral epidural fluid collection had resolved.

\section{Discussion}

The clinical and imaging features of spontaneous intracranial hypotension (hypovolemia) have become increasingly recognized in the past 15 years. ${ }^{2}$ Most of these cases are associated with abnormal epidural fluid collections of varying sizes along 

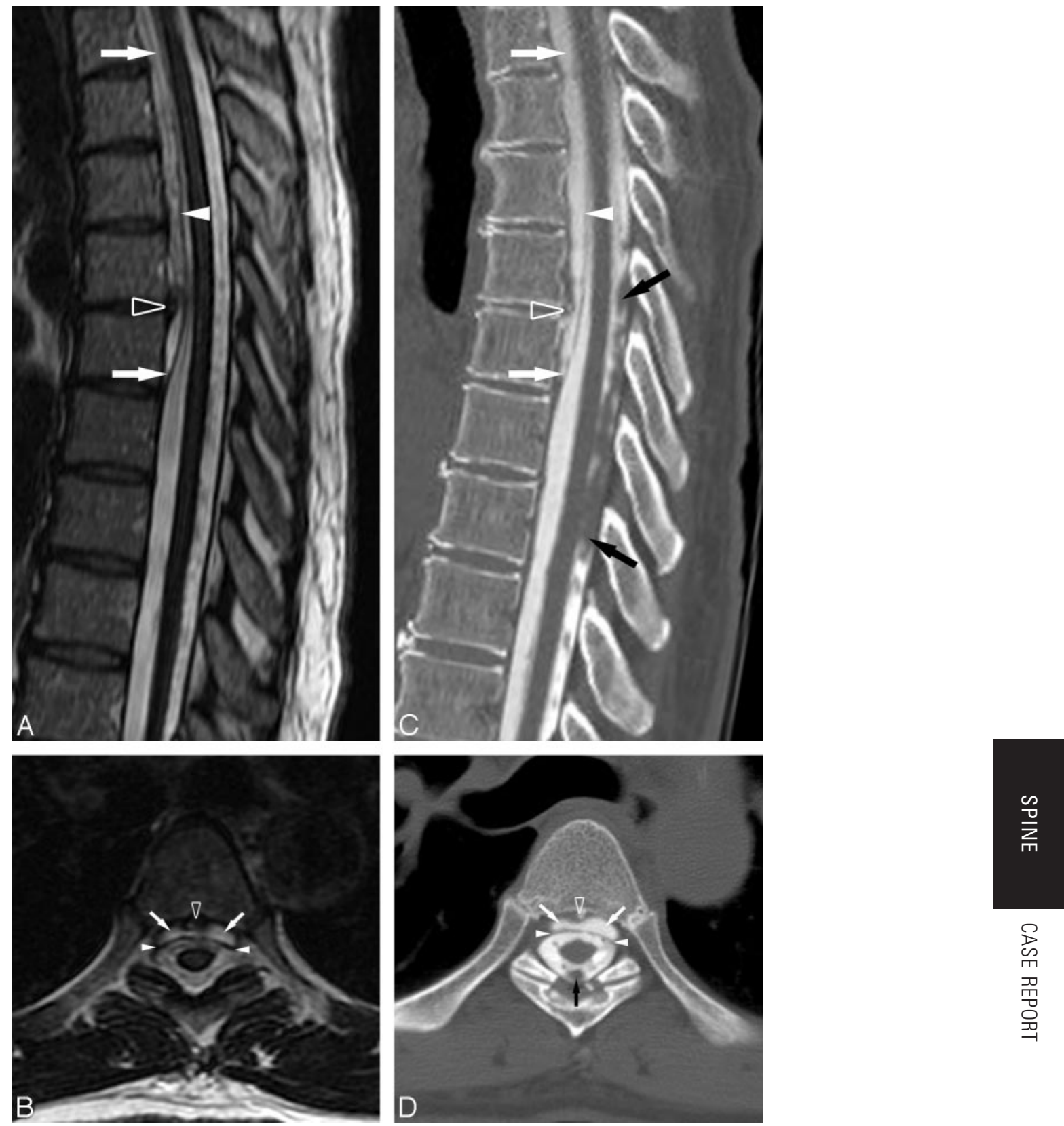

Fig 1. A, Sagittal T2-weighted (TR, $3300 \mathrm{msec}$; TE, $100.8 \mathrm{msec}$ ) image of the thoracic spine defines the caudal aspect of the epidural fluid collection at T6-T7. A prominent central disk extrusion is present at T5-T6. B, Axial T2-weighted (TR, $2600 \mathrm{msec}$; TE, $103.1 \mathrm{msec}$ ) image through the T5-T6 disk extrusion further delineates the ventral epidural fluid collection displacing the dura posteriorly. Note the prominent T2 hypointensity on the cord surface secondary to superficial siderosis. $C, D$, Postmyelography CT images in similar planes and at analogous levels to the MR images demonstrate that the ventral epidural fluid collection is opacified by intrathecal contrast to a degree similar to CSF within the thecal sac, confirming the presence of an active CSF leak. The prominent T5-T6 disk extrusion is partially calcified. Ventral epidural fluid collection (white arrows), dura (white arrowheads), T5-T6 disk extrusion (open arrowhead), and subarachnoid clot (black arrows) are shown.

the spinal axis secondary to a CSF leak. The dural tear causing the leak, often related to focal intervertebral disk extrusions or friable perineural cysts, is most commonly near the cervicothoracic junction or in the mid to upper thoracic spine. More recently, it has become recognized that a subset of patients with this pattern of spinal dural tear and epidural fluid collection manifest imaging and clinical features of superficial siderosis. ${ }^{3}$ Friable vessels at the site of the dural tear are the likely source of chronic bleeding into the subarachnoid space, leading to superficial siderosis. In these instances, surgical correction of the dural tear is necessary and holds hope to prevent progressive neurologic deficits. For facilitation of surgery, precise preoperative localization of the dural tear is needed.

The use of image subtraction during myelography with both manual and digital techniques was remotely described but never achieved widespread application, ${ }^{4,5}$ likely related to the adoption of postmyelography CT. Some limited applications of similar methodology have been reported. Digital subtraction cisternography has been reported as a useful technique to identify intracranial CSF fistulas. ${ }^{6-8}$ More recently, digital subtraction myelography was successfully used to localize the dural tear in the case of a high cervical postoperative pseudomeningocele. ${ }^{9}$ 

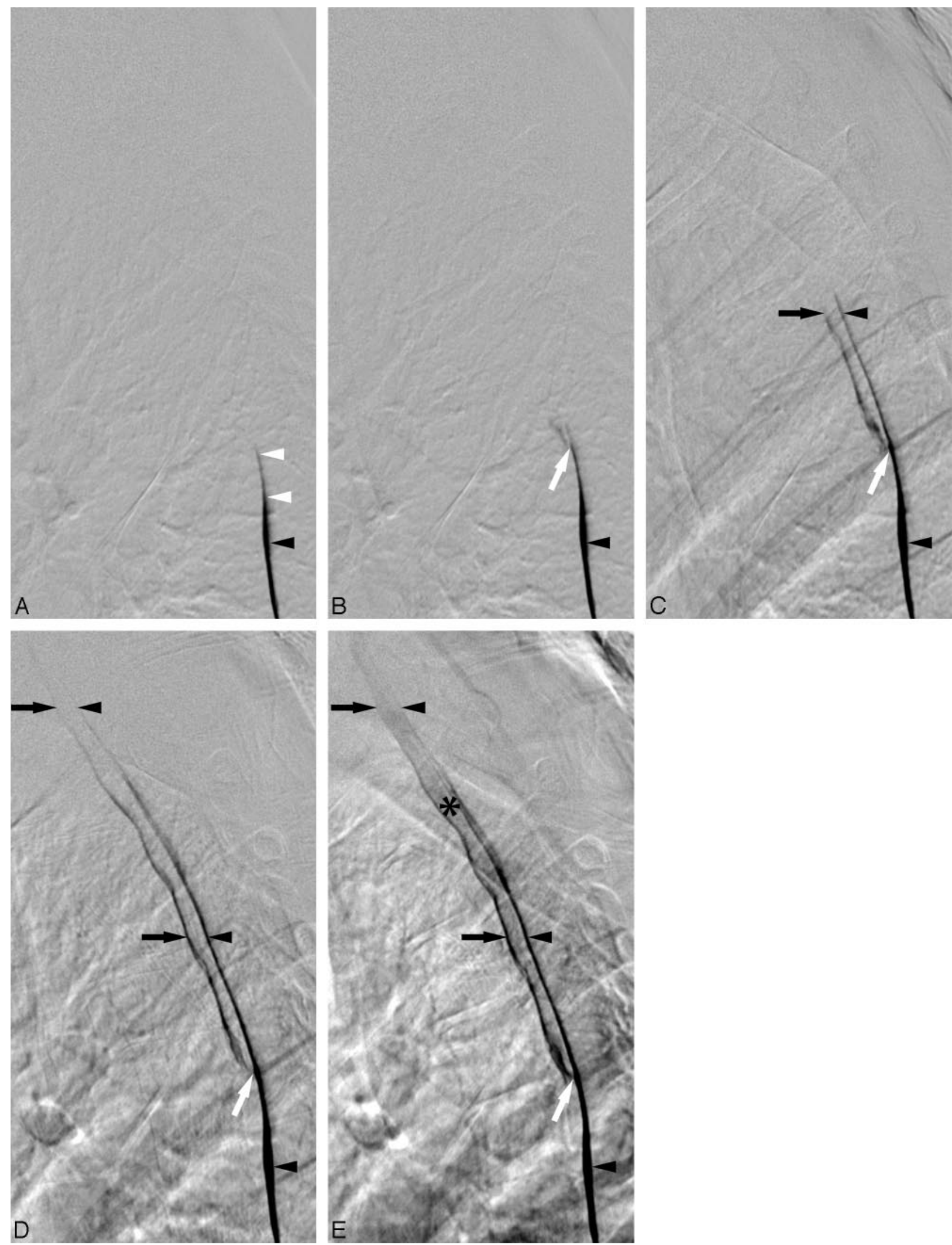

Fig 2. Lateral views of the thoracic spine acquired during digital subtraction myelography with the patient in the prone position. For temporal reference, the initial image is designated as time of $(A) 0.0$ second, and the subsequent images are at $(B) 0.4$ second, $(C) 1.4$ seconds, $(D) 3.6$ seconds, and $(E) 11.2$ seconds. Contrast is seen to progressively extend cephalad dependently within the thecal sac (black arrowheads). At T6-T7, the contrast slightly deviates dorsally because of mass effect on the dura by the caudal aspect of the epidural fluid collection (white arrowheads). Contrast focally extravasates through the ventral dural tear at T5-T6 (white arrow) into the epidural collection (black arrows). Contrast extends cephalad within both the thecal sac and the epidural collection. Ultimately, the epidural fluid collection becomes more dense (*) because of its smaller volume relative to the subarachnoid space. 
The MR imaging features of spinal dural tears have been described, with one of the most prominent imaging findings being an abnormal epidural fluid collection. ${ }^{10}$ When patients have characteristic findings on MR imaging, we typically proceed to myelography followed by postmyelography CT that encompasses the entire extent of the abnormal epidural fluid collection. This typically provides unequivocal confirmation of an active CSF leak and often allows identification of the dural tear. If precise localization is not possible, findings associated with spontaneous CSF leaks such as perineural cysts or focal disk extrusions may be identified, and the region of interest is able to be further narrowed. If needed, dynamic CT myelography ${ }^{1}$ can then be performed through a smaller number of vertebral levels (to reduce radiation exposure to the patient). In the current case, the presence of a prominent, partially calcified disk extrusion at T5-T6 was felt to be the most likely candidate for the location of the CSF fistula, though the patient did have focal disk pathologic features at other levels. It is unfortunate that on the initial acquisition of the upper thoracic spine during dynamic CT myelography, a significant amount of contrast was already present in the ventral epidural fluid collection spanning 5 vertebral levels. The rapidity of the CSF leak in this case was well demonstrated by the digital subtraction myelogram in that it only took several seconds from the initial contrast extravasation at T5-T6 until contrast reached the cervicothoracic junction (Fig 2).

\section{Conclusions}

Digital subtraction myelography is a useful tool as an adjunct to the more commonly accepted imaging techniques to evaluate patients with spontaneous spinal CSF leaks, particularly those that are quite rapid. The case of this patient also further underscores that dural tears can be a source of chronic subarachnoid hemorrhage and may lead to superficial siderosis.

\section{References}

1. Luetmer PH, Mokri B. Dynamic CT myelography: a technique for localizing high-flow spinal cerebrospinal fluid leaks. AJNR Am J Neuroradiol 2003;24:1711-14

2. Mokri B. Low cerebrospinal fluid pressure syndromes. Neurol Clin 2004;22: 55-74, vi

3. Kumar N, Cohen-Gadol AA, Wright RA, et al. Superficial siderosis. Neurology 2006;66:1144-52

4. El Gammal T. Cervical myelography and posterior fossa examinations with Amipaque: use of magnification and subtraction. Radiology 1980;136:219-22

5. Sherry RG, Anderson RE. Real-time digitally subtracted fluoroscopy for cervical myelography. Radiology 1984;151:243-44

6. Takahashi T, Mutsuga N, Aoki T, et al. Localization of dural fistulas using metrizamide digital subtraction fluoroscopic cisternography. J Neurosurg 1988;68:721-25

7. Byrne JV, Ingram CE, MacVicar D, et al. Digital subtraction cisternography: a new approach to fistula localisation in cerebrospinal fluid rhinorrhoea. J Neurol Neurosurg Psychiatry 1990;53:1072-75

8. Wakhloo AK, van Velthoven V, Schumacher M, et al. Evaluation of MR imaging, digital subtraction cisternography, and CT cisternography in diagnosing CSF fistula. Acta Neurochir (Wien) 1991;111:119-27

9. Phillips CD, Kaptain GJ, Razack N. Depiction of a postoperative pseudomeningocele with digital subtraction myelography. AJNR Am J Neuroradiol 2002;23:337-38

10. Rabin BM, Roychowdhury S, Meyer JR, et al. Spontaneous intracranial hypotension: spinal MR findings. AJNR Am J Neuroradiol 1998;19:1034-39 